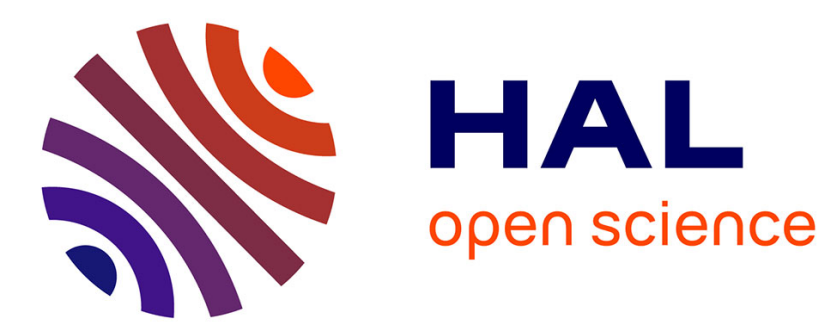

\title{
Hybrid nanostructured plasmonic electrodes for flexible organic light-emitting diodes
}

Shiwei Wu, Yilian Li, Hong Lian, Gaëtan Lévêque, B. Grandidier, Pierre-Michel Adam, Davy Gérard, Renaud Bachelot, Tao Xu, Bin Wei

\section{- To cite this version:}

Shiwei Wu, Yilian Li, Hong Lian, Gaëtan Lévêque, B. Grandidier, et al.. Hybrid nanostructured plasmonic electrodes for flexible organic light-emitting diodes. Nanotechnology, 2020, 31 (37), pp.375203. 10.1088/1361-6528/ab94df . hal-03418469

\author{
HAL Id: hal-03418469 \\ https://hal.science/hal-03418469
}

Submitted on 7 Nov 2021

HAL is a multi-disciplinary open access archive for the deposit and dissemination of scientific research documents, whether they are published or not. The documents may come from teaching and research institutions in France or abroad, or from public or private research centers.
L'archive ouverte pluridisciplinaire HAL, est destinée au dépôt et à la diffusion de documents scientifiques de niveau recherche, publiés ou non, émanant des établissements d'enseignement et de recherche français ou étrangers, des laboratoires publics ou privés. 


\section{Hybrid nanostructured plasmonic electrodes for flexible organic light-emitting diodes}

Shiwei $\mathrm{Wu}^{1}$, Yilian $\mathrm{Li}^{1}$, Hong Lian ${ }^{1}$, Gaëtan Lévêque ${ }^{2}$, Bruno Grandidier ${ }^{2}$, PierreMichel Adam ${ }^{3}$, Davy Gérard ${ }^{3}$, Renaud Bachelot ${ }^{3}$, Tao $\mathrm{Xu}^{1 *}$, Bin Wei $^{{ }^{*}}$

${ }^{1 .}$ School of Mechatronic Engineering and Automation, Key Laboratory of Advanced Display and System Applications, Ministry of Education, Shanghai University, 200072, Shanghai, China

2. IEMN-UMR8520, CNRS, Univ. Lille, Centrale Lille, ISEN, Univ. Valenciennes, F59000 Lille, France

${ }^{3 .}$ Light, nanomaterials, nanotechnologies (L2n), CNRS ERL 7004, University of Technology of Troyes, F-10004 Troyes Cedex, France

Corresponding Author

Email: xtld@shu.edu.cn, bwei@shu.edu.cn 


\begin{abstract}
Improved performance in flexible organic light-emitting diodes (OLEDs) is demonstrated by using a hybrid nanostructured plasmonic electrode consisting of silver nanowires (AgNWs) decorated with silver nanoparticles (AgNPs) and covered by exfoliated graphene sheets. Such all-solution processed electrodes show high optical transparency and electrical conductivity. When integrated in an OLED with super yellow polyphenylene vinylene as the emissive layer, the plasmon coupling of the NWNP hybrid plasmonic system is found to significantly enhance the fluorescence, demonstrated by both simulations and photoluminescence measurements, leading to a current efficiency of $11.61 \mathrm{~cd} \mathrm{~A}^{-1}$ and a maximum luminance of $20008 \mathrm{~cd} \mathrm{~m}^{-2}$ in OLEDs. Stress studies reveal a superior mechanical flexibility to the commercial indium-tinoxide (ITO) counterparts, due to the incorporation of exfoliated graphene sheets. Our results show that these hybrid nanostructured plasmonic electrodes can be applied as an effective alternative to ITO for use in high-performance flexible OLEDs.
\end{abstract}

Keywords: flexible organic light-emitting diodes, flexible transparent electrode, surface plasmon, nanowire-nanoparticle junction, exfoliated graphene 


\section{Introduction}

Flexible Organic Light-Emitting Diodes (OLEDs) have attracted considerable attention for use in next-generation foldable or roll type applications due to their advantages such as low-cost and large-area fabrication using solution processing. ${ }^{[1,2]}$ One key issue involving flexible devices is to develop novel Flexible Transparent Electrodes (FTEs) which need to demonstrate superior electrical, optical and mechanical properties. So far, indium-tin-oxide (ITO) is the most commercially used transparent electrode, but its role in flexible optoelectronics is limited due to the inherent shortcomings of rigidity and brittleness. ${ }^{[3-5]}$ Various types of FTEs have been proposed to replace ITO such as: metallic nanostructures, ${ }^{[6-9]}$ carbon-based materials, ${ }^{[10,11]}$ conducting polymers ${ }^{[12-14]}$ and hybrid films. ${ }^{[15,16]}$ Among them, silver nanowires (AgNWs) is one of the most promising candidates as an alternative FTE for flexible devices because of the attractive mechanical flexibility, ${ }^{[17,18]}$ excellent conductivity and good optical transmittance. ${ }^{[19,20]}$ Nevertheless, some drawbacks remain to be solved for AgNWs-based FTEs. First, the randomly distributed $\mathrm{AgNWs}$ fabricated by solution processing can result in large surface roughness whose structures may penetrate through the active layer, leading to short circuits of the thin-film devices. ${ }^{[21-24]}$ Second, percolation of charges through junctions between AgNWs can lead to large contact resistance. ${ }^{[25,26]}$ In addition, the nanowire junctions may get damaged after large tensile deformation. In order to circumvent these issues, mechanical pressing and thermal annealing of the polymer matrix that contains the AgNWs have been applied to drastically reduce the surface roughness of the layer. ${ }^{[27,28]}$ Another method consists of the deposition of twodimensional graphene layers onto the network of $\mathrm{AgNW}$ s to enhance the mechanical stability of FTEs, taking advantage of the excellent stretchability of graphene. ${ }^{[29]}$ In addition, this scheme protects the AgNWs from getting oxidized, improving the longterm electrical stability of the FTEs. ${ }^{[30]}$

Despite the improved mechanical/chemical stability of FTEs combining AgNWs and graphene sheets, the efficiency of the flexible OLEDs still falls behind that of their ITObased counterparts. One strategy is to use plasmonic metal nanostructures which 
support surface plasmon that can couple with excitons to enhance light extraction in OLEDs. ${ }^{[31]}$ Surface plasmons are collective oscillations of free electrons in a metal at an interface with a dielectric, which can result in electronic and photonic resonances, strong absorption and light scattering, and an enhancement of the local electromagnetic fields. ${ }^{[32]}$ In particular, the overlap of local electromagnetic field of the excitons in the emissive layer and surface plasmons leads to the coupling effect between excitons and surface plamons which conduce to significant radiative emission through effective energy transfer in OLEDs. ${ }^{[33]}$ Among various metal nanostructures, silver nanoparticles (AgNPs) are considered as promising plasmonic materials due to the localized surface plasmon resonance (LSPR) in the visible and high scattering efficiency. ${ }^{[34]}$ The application of AgNPs in OLEDs has shown to enhance device performance. ${ }^{[35,36]}$ This effect is even enhanced when AgNPs are coupled with AgNWs to constitute a hybrid plasmonic system. ${ }^{[37]}$ AgNPs couple the incident light into surface plasmon polaritons (SPP) on the surface of AgNW. Such a plasmon coupling at NW-NP junctions induces light confinement and local electrical field enhancement larger than they would in AgNWs or AgNPs alone, which improves resonant coupling to excitons in the organic active layer. ${ }^{[38,39]}$ It is thus interesting to develop novel plasmonic FTEs containing different hybrid metallic nanostructures to improve the performances of flexible OLEDs.

In this work, we demonstrate a promising FTE based on hybrid nanostructures which takes advantage of both the plasmon effect of AgNWs:AgNPs and the excellent flexibility of exfoliated graphene (EG). Guided by electromagnetic numerical simulations of the plasmon effect in NW-NP junctions, we first show that the AgNWs:AgNPs systems act as efficient nanoantennas and nanoresonators, enhancing the fluorescence of the emissive layer in the FTE. Adding EG results in a FTE with a superior mechanical stability, a low sheet resistance of $22 \Omega \mathrm{sq}^{-1}$ and a high optical transmittance of $80 \%$. When applied in flexible OLEDs using super yellow polyphenylene vinylene (SY-PPV) as emissive material, it yields a current efficiency of $11.6 \mathrm{~cd} \mathrm{~A}^{-1}$ and a luminance of $20008 \mathrm{~cd} \mathrm{~m}^{-2}$. Notably, the flexible devices can maintain 
$90 \%$ of their initial luminescence after 1000 bending iterations, outperforming their ITO counterparts.

\section{Experimental Section}

\section{Materials}

The solution of AgNPs (60 $\mathrm{nm}$ in diameter) was purchased from Sigma Aldrich. The solution of AgNWs (80 nm in diameter and 20-30 $\mu \mathrm{m}$ in length) was ordered from BlueNano Company (Cornelius, NC, USA). EG layers dispersed in N-Methyl pyrrolidone solution at a concentration of $4 \mathrm{mg} \mathrm{mL}^{-1}$ was purchased from XFNANO (Nanjing, China). The lateral size of EG was estimated to be $0.5-3 \mu \mathrm{m}$, while the thickness was measured to be 0.3-2 nm. Super Yellow (SY) was purchased from Xi 'an Polymer Light Technology Corp. Poly (3, 4-ethylenedioxy-thiophene) doped with polystyrene sulfonate (PEDOT:PSS) aqueous solution of Clevios PVP 4083 were purchased from Heraeus, Germany. The small-molecular material Liq was obtained from e-Ray Optoelectronics Corp. Chlorobenzene and 1,8-diiodooctane were ordered from Sigma Aldrich.

\section{Fabrication of hybrid nanostructured FTEs}

The AgNWs:AgNPs/EG FTEs were prepared by a successive solution process on precleaned Polyethylene terephthalate (PET) substrates. The PET substrate was cleaned sequentially in an ultrasonic bath with detergent, deionized water and isopropanol. AgNPs and AgNWs mixed solution (AgNPs: AgNWs =1:10) was then deposited on the PET substrate followed by an annealing at $110{ }^{\circ} \mathrm{C}$ for $15 \mathrm{~min}$. Finally, EG dispersion was sprayed onto the top of the AgNPs:AgNWs layer by an airbrush system. The thickness of the top EG layer was controlled by tuning the spraying height and time. The EG dispersion was first atomized into small droplets by toggling the air valve and then annealed at $110{ }^{\circ} \mathrm{C}$ for $1 \mathrm{~min}$.

\section{Fabrication of flexible OLEDs}

OLEDs using ITO, AgNWs: AgNPs and AgNWs: AgNPs/EG based electrodes were 
fabricated. Prior to the film deposition, the ITO-coated glass substrates and PET substrates were thoroughly cleaned successively in an ultrasonic bath with detergent, deionized water and acetone for $20 \mathrm{~min}$ each, and then dried using a pure nitrogen gas stream. The OLED device is composed of PEDOT:PSS with a thickness of $40 \mathrm{~nm}$ as the hole transporting layer, $60 \mathrm{~nm}$ of SY-PPV as the emitting layer, $1 \mathrm{~nm}$ of Liq as the electron injection layer, and $100 \mathrm{~nm}$ of $\mathrm{Al}$ as the cathode. PEDOT:PSS was spin-coated separately on the prepared ITO and various types of FTEs/PET substrates at $3000 \mathrm{rpm}$ for $60 \mathrm{~s}$. Then, it was annealed at $110{ }^{\circ} \mathrm{C}$ for $30 \mathrm{~min}$. For the emitting layer in OLEDs, SY-PPV was dissolved in chlorobenzene at a concentration of $20 \mathrm{mg} \mathrm{mL}^{-1}$. The solution was spin-coated onto ITO or FTE/PEDOT:PSS at $1000 \mathrm{rpm}$ for $60 \mathrm{~s}$. Finally, the substrate was transferred into high vacuum chamber at a base pressure of about $10^{-6} \mathrm{~Pa}$ for evaporation. The evaporation rates of Liq and Al were about $0.1 \AA \mathrm{s}^{-1}$ and $5 \AA \mathrm{s}^{-1}$, respectively. A quartz-crystal monitor was used to monitor the deposition rate and film thickness. The active area defined by the overlap of the anode and the Al cathode was $2 \mathrm{~mm} \times 2 \mathrm{~mm}$.

\section{Characterization}

The surface morphology of hybrid nanostructured FTEs was analyzed with scanning electron microscopy (SEM, HITACHI S-4800) and atomic force microscopy (AFM, Nanonavi SPA-400SPM). The optical transmittances and the sheet resistances $\left(R_{s}\right)$ of FTEs were measured with a UV-vis spectrophotometer (HITACHI Ue3900H) and 4point probe system (RS8, BEGA Technologies, Korea), respectively. The photoluminescence (PL) spectra and time-resolved PL spectra were measured with a FLSP 920 fluorescence spectrometer. Raman spectroscopy was carried out using a Horiba LabRam spectrometer with a laser operating at $473 \mathrm{~nm}$ wavelength as an excitation source. The current density voltage-luminance characteristics and electroluminescence spectra of the corresponding OLED devices were characterized simultaneously by a computer-controlled programmable Keithley model 2400 power source and a PhotoResearch PR650 spectrometer. The spectra were acquired along the normal angle of incidence. All the measurements were performed in air under ambient 
conditions without device encapsulation.

\section{Electromagnetic simulation}

Absorption spectra and distributions of the electric near-field amplitude of a single $\mathrm{AgNW}$, having a diameter of $80 \mathrm{~nm}$ and a length of $3.5 \mu \mathrm{m}$, or an $\mathrm{AgNW}$ decorated with $60 \mathrm{~nm}$-sized spherical AgNPs were calculated using the Finite Difference Time Domain (FDTD) method (Comsol Multiphysics software). ${ }^{[40]}$ In the calculation, we considered planewaves propagating from the air, with a normal incidence to the AgNW/glass substrate or AgNW:AgNPs/glass substrate, and polarized parallel to the AgNW's axis. The dielectric constant of silver has been taken from Ref. 41, while the refractive index of glass is 1.5 . It should be noted that a thin native oxide layer with a thickness of $1 \mathrm{~nm}$ was considered around the AgNW:AgNP system. Perfectly matched layers were applied around the simulation domain and symmetries were used to optimize memory and computation time.

\section{Results and discussion}

In order to get a first insight into the plasmonic effect induced by the hybrid metal nanostructures, numerical simulations were performed on NW-NP plasmon couplings. Calculations were carried out on a $3.5 \mu \mathrm{m}-$ long AgNW. We studied the effect of NWNP plasmon coupling on three different structures that are believed to be characteristic of those usually experimentally observed: bare AgNW, AgNW with 8 separated AgNPs and 5 clusters of AgNPs. Figures 1(a) shows calculated distributions of the electric nearfield amplitude for the three cases. The corresponding absorption spectra and scattering spectra are shown in Figure 1(b) and (c), respectively. In Figure 1(a), the chosen wavelengths correspond to strong resonances in Figure 1(b). Without AgNPs (a1 case), propagating surface plasmons are launched, travel back and forth along the AgNW and interfere with each other, resulting in a stationary mode along the AgNW, as confirmed by the field distribution that shows a typical longitudinal oscillating pattern. ${ }^{[42]}$ As shown in the corresponding spectra of Figure 1(b) and (c), this resonance can occur at many wavelengths when NW length $\mathrm{L}=\mathrm{p} \lambda / 2 \mathrm{n}_{\text {eff }}$, where $\mathrm{n}_{\text {eff }}$ is the effective index of the 
plasmon monde and $\mathrm{p}$ is an integer. In the presence of AgNPs (a2 case), strong resonances are obtained (Figure 1(b)) and it is seen that the field magnitude for the NWNP plasmon coupling effect is related to the arrangement of AgNPs. Compared to Figure 1(a)-a1, Figure 1(a)-a2 shows localized electromagnetic hot spots which are associated to a strengthening of the local density of states (LDOS) and the associated spectral features. With clusters of AgNPs (a3 case), the local electromagnetic field is strongly enhanced and the hot spots are numerous, suggesting a much higher photonic density of state. It should be noted that this effect is likely to result from a coupling between the propagating surface plasmon supported by the AgNW and the localized surface plasmons supported by either single AgNP or clusters of AgNPs. For example, AgNP and clusters can resonantly launch and drive the propagative plasmons. In turn, the propagating surface plasmons can excite the localized plasmons modes of the AgNP and clusters. As a result, NP-NW resonant gap modes are strongly excited. In Figure 1(a)-a3, it is worth noticing that strong oscillations appear between clusters and AgNW and between different clusters, suggesting local Fabry Perot cavities and significant contribution of propagating surface plasmons. This could be due to the fact that, compared to the single AgNP, the complex structure of cluster is associated to a much richer angular spectrum of wave vectors that are susceptible to well match the dispersion function of the propagating surface plasmons.

In Figure 1(b), it turns out that combination of AgNW and clusters of AgNPs leads to a strong enhancement absorption over a broad spectral range (300-700 nm). The scattering spectrum is also broadly enhanced, as it is shown in Figure 1(c). When the hybrid plasmonic nanostructures were integrated into the OLED device, the absorption is likely to play an important role in the near-field plasmon-exciton coupling within OLEDs, while the scattering properties are of prime importance because we aim at enhancing the far-field emission of the OLEDs. As a conclusion of this part, the FDTD simulations qualitatively demonstrate that the plasmon effect of the NW-NP hybrid system can significantly enhance the electric field intensity around the junctions as well as spectral properties. Such an enhancement will be essential to increase the radiative 
emission of the OLEDs when AgNWs:AgNPs are integrated in the transparent electrode.

(a)
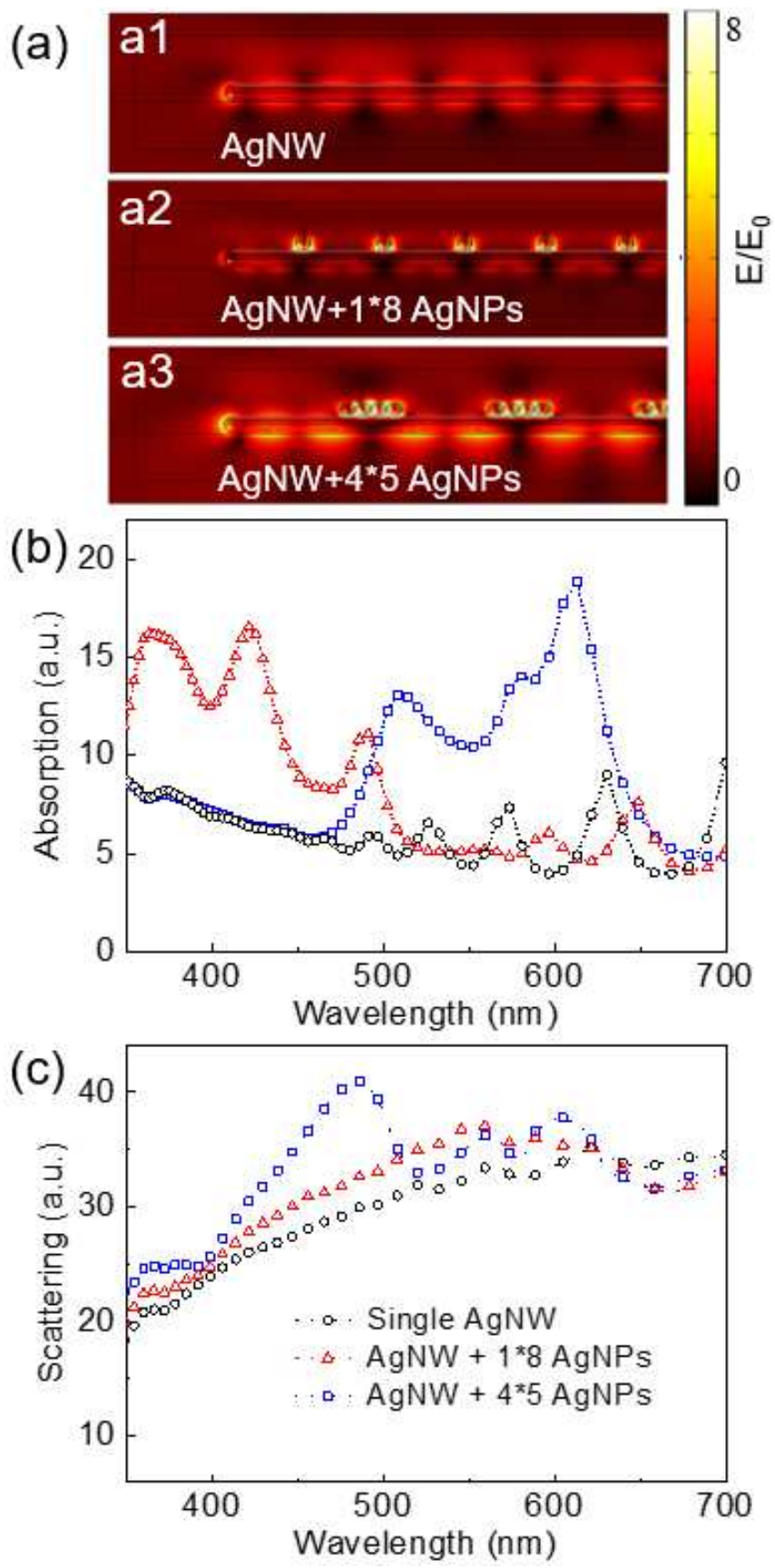

Figure 1. FDTD electromagnetic simulation on a 3.5 $\mu \mathrm{m}$-long AgNW coupled with AgNPs. (a) Map of the magnitude of the electric near-field, in three different cases. a1: without AgNPs $(\lambda=630 \mathrm{~nm})$, a2: with 8 separated AgNPs $(\lambda=425 \mathrm{~nm})$, a3: with 5 clusters of AgNPs $(\lambda=610 \mathrm{~nm})$. The magnitude of the enhanced electric field intensity is indicated by the color scale. (b) Absorption and (c) scattering spectra for the same three cases. 
In the second part of this work, the hybrid nanostructured FTEs were fabricated according to the iterative processes illustrated in Figure 2(a). First, AgNWs:AgNPs were spin-coated on the PET substrate. The morphology of the AgNW network coupled with AgNPs was observed with SEM and AFM, as shown in Figures 2(b) and (c). AgNWs with a diameter of $80 \mathrm{~nm}$ were randomly distributed and overlapped with each other on the surface. AgNPs with a diameter of $60 \mathrm{~nm}$ were found not only to fill up the empty gaps in the network of AgNWs, but also to couple with the AgNWs forming the NW-NP junctions. In some areas highlighted by circles, clusters AgNPs are visible. Therefore, although the distribution of AgNWs:AgNPs is not well controlled, both configurations considered in the FDTD simulations above do exist and should have an impact on the optical properties of the transparent electrode.
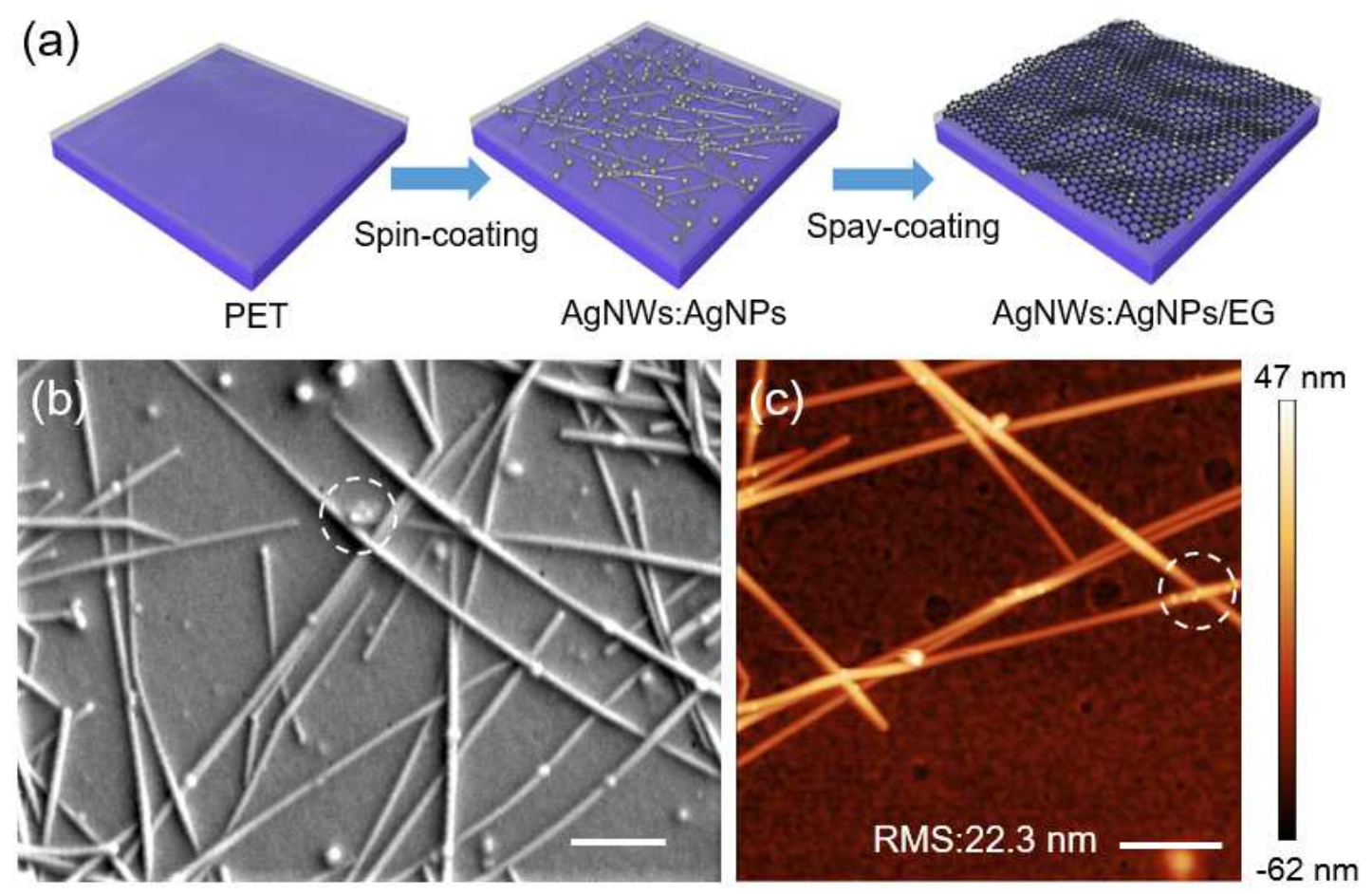

Figure 2. (a) Schematic of the fabrication process of hybrid nanostructured FTEs. (b) SEM and (c) AFM images of AgNWs:AgNPs. The scale bar is $500 \mathrm{~nm}$. The white circles indicate regions of high concentrations of Ag NPs.

To experimentally determine the degree of coupling between the surface plasmons of the AgNW:AgNP electrode and the excitons of the emissive layer, PL and time-resolved PL measurements were conducted. SY and PEDOT:PSS, which were used as the 
fluorescent emissive layer and hole transport layer, were deposited on the PET substrates with AgNWs:AgNPs. Figure 3(a) shows the steady-state PL spectra for samples consisting of PEDOT:PSS/SY, AgNWs/PEDOT:PSS/SY and AgNWs:AgNPs/PEDOT:PSS/SY. As a control, PEDOT:PSS/SY yielded the lowest PL intensity because of significant exciton quenching at the PEDOT:PSS/SY interface. ${ }^{[43]}$ The intensity was increased by $\sim 25 \%$ when AgNWs were added, while the AgNWs:AgNPs/PEDOT:PSS/SY films demonstrated the highest PL intensity, consistent with our predictions. The time-resolved PL measurements for PEDOT:PSS/SY, AgNWs/PEDOT:PSS/SY and AgNWs:AgNPs/PEDOT:PSS/SY are plotted in Figure 3(b). A significant increase in the PL decay rate was observed when AgNWs:AgNPs were added, leading to the decrease in the lifetime of the exciton. From the decay values, the average PL lifetime was calculated to be $0.90 \mathrm{~ns}$ for PEDOT:PSS/SY, $0.81 \mathrm{~ns}$ for AgNWs/PEDOT:PSS/SY and $0.72 \mathrm{~ns}$ for AgNWs:AgNPs/PEDOT:PSS/SY, respectively.
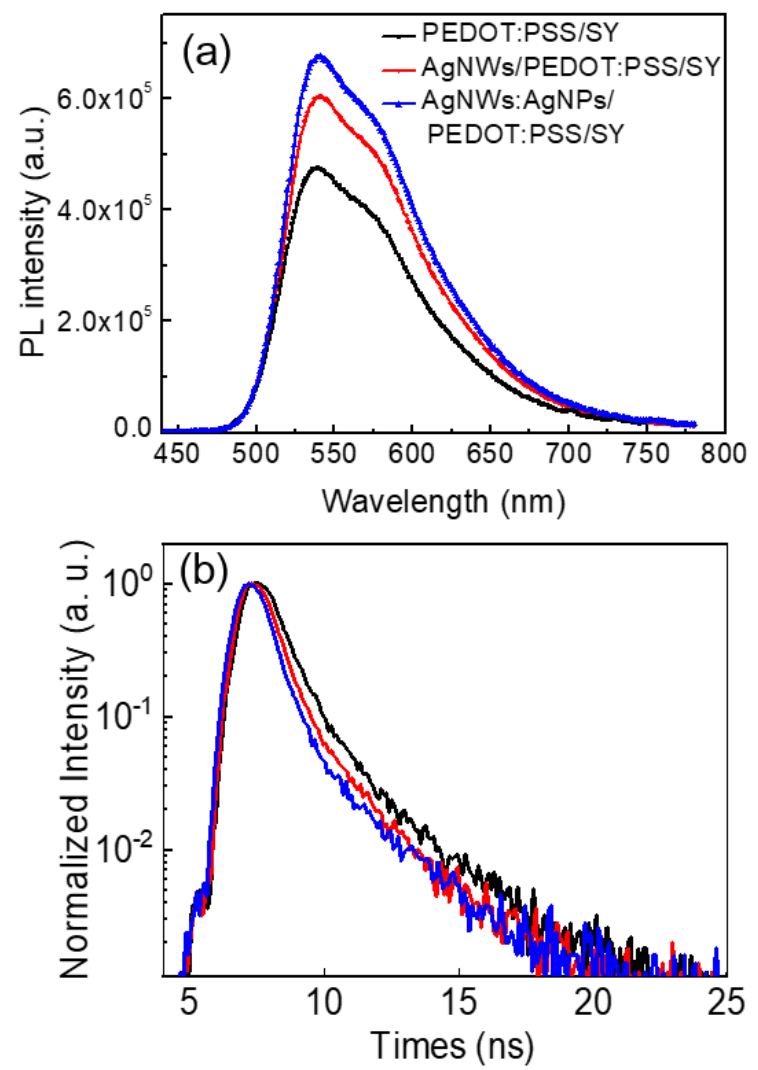

Figure 3. (a) PL spectra and (b) Transient PL decay curves of PEDOT:PSS/SY, AgNWs/PEDOT:PSS/SY and AgNWs:AgNPs/PEDOT:PSS/SY. 
The mechanism of the typical coupling process is given in the schematic diagram of Figure 4(a). First, the excitons are generated in the emissive layer which can decay either radiatively or nonradiatively. Then, the excitons get coupled with the hybrid AgNW:AgNPs surface plasmons modes (described in the above "electromagnetic simulation" section) that have a high density of LDOS. Finally, this coupled excited state gets radiatively de-excited through plasmon-enhanced emission. Such coupling process is much faster than the spontaneous recombination of excitons, ${ }^{[44]}$ leading to the reduction in the lifetime of excitons supported by the time-resolved PL measurements. It should be stressed that this fast plasmon coupling minimizes the unwanted non-radiative recombination. Furthermore, it is known that the effective surface plasmons extend away from the surface of the metal nanostructures by 10 to 70 nm. ${ }^{[45]}$ In our case, AgNWs:AgNPs were separated by $40 \mathrm{~nm}$ from the emissive layer, a distance small enough to that the plasmons efficiently assist the coupling of the exciton in the emissive layer. Finally, our AgNWs:AgNPs hybrid systems effectively combine both localized surface plasmons and propagating surface plasmon polaritons, leading to a stronger hybrid plasmon coupling, demonstrated by both simulations and PL measurements. Hence, these results indicate the existence of plasmon coupling effect in the hybrid nanostructured FTEs, which can further enhance the performances of OLEDs.

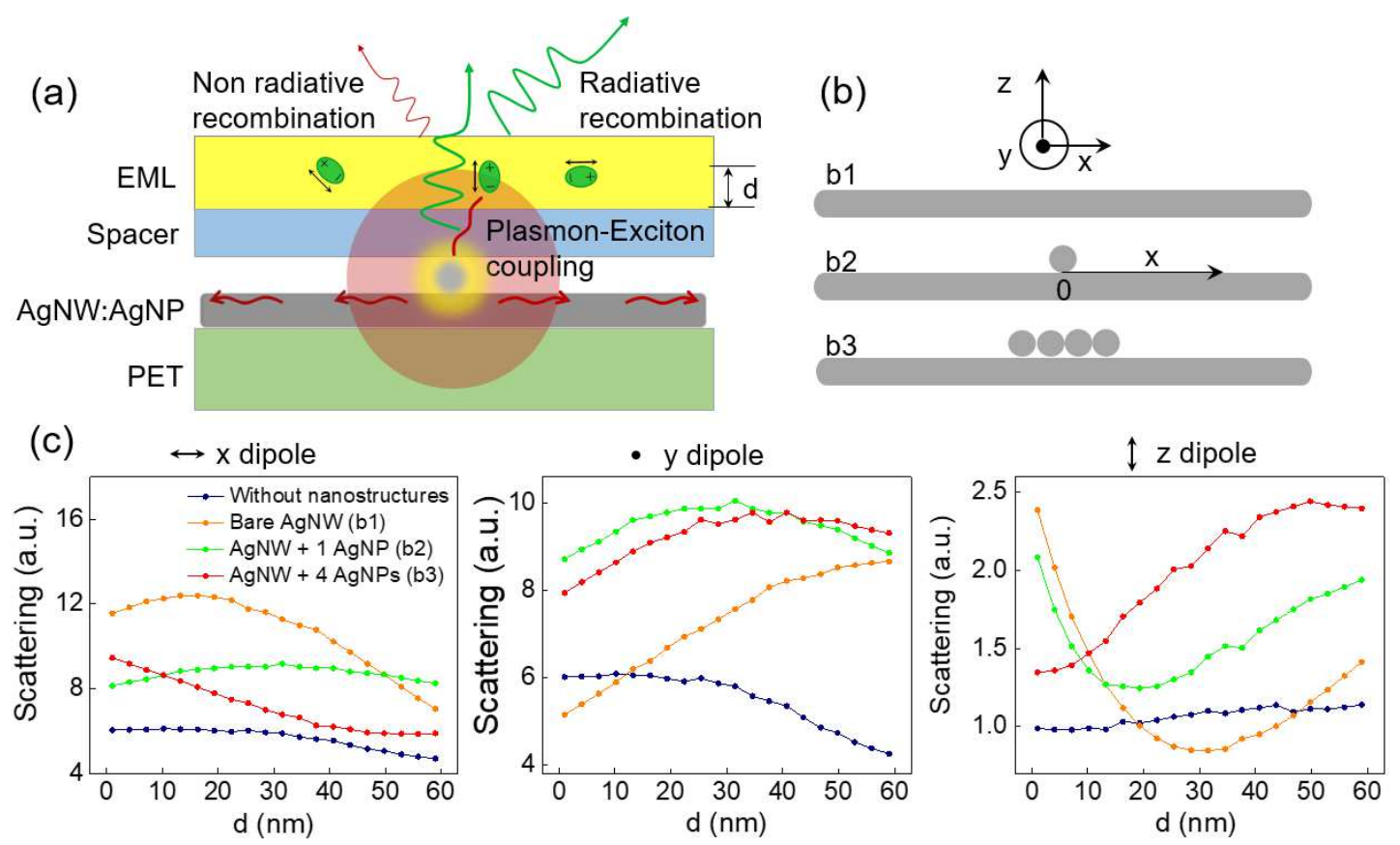


Figure 4. (a) Schematic diagram showing the coupling mechanism between the surface plasmons of AgNW:AgNP and the excitons. Within the emitting layer (EML), different dipole orientations are represented. $\mathrm{d}$ is the distance between a dipole and the top surface of the layer used as a spacer. (b) Geometries of the plasmonic nanostrutures considered in the simulation. b1: bare AgNW, b2: AgNW coupled with one AgNP, b3: AgNW coupled with four AgNPs. (c) Scattering cross section in air (corresponding to the actual light output channel of OLED) calculated for three different dipole orientations: $\mathrm{x}$ (along the NW axis), $\mathrm{y}$ (in-plane, perpendicular to the NW axis) and $\mathrm{z}$ (out-of-plane) with different geometries of the plasmonic nanostructures.

In order to get a deep understanding on the process of enhancement of light emission observed in Figure 3, additional FDTD calculations were carried out. The realistic geometry illustrated in Figure 4(a) was considered. A light-emitting exciton produced by charge injection within the 60 -nm thick emitting layer (EML) was represented by a point-like emitted dipole $(\lambda=550 \mathrm{~nm})$ whose spatial orientation and position is a priori random in a real device. Three geometries of the plasmonic nanostrutures were considered: bare AgNW (Figure 4(b)-b1), AgNW:AgNP (Figure 4(b)-b2), AgNW: AgNP-cluster (Figure 4(b)-b3). The dipole was placed at the middle of the structure $(x=0, y=0)$, within the emissive layer, at the distance $d$ ranging from 0 to $60 \mathrm{~nm}$ on the top of the 40-nm thick spacer (see Figure 4(a)). The scattering cross section in air (corresponding to the actual light output channel of OLED) was calculated for three different dipole orientations: $\mathrm{x}$ (along the NW axis), y (in-plane, perpendicular to the NW axis) and z (out-of-plane). Figure 4(c) shows the results of the calculation. For comparison, dipole emission without any plasmonic nanostructures was considered as reference which corresponds to the bare OLEDs. In general, scattering efficiency turns out to get improved by the presence of the plasmonic nanostructures. It is worth noticing that the different plasmonic nanostructures play different role relative to the dipole orientation. X-dipole emission is mainly boosted by the bare AgNW, which is due to the fact that $\mathrm{x}$ dipoles, especially at short distance from the AgNW, can get easily coupled with the propagative surface plasmons. Z-dipoles are very sensitive to the 
AgNW: AgNP-cluster which is a complex structure susceptible to generate rich out-ofplane LDOS. The y dipole emission benefits from both AgNW:AgNP and AgNW:AgNP-cluster systems. While the scattering efficiency without nanostructures is poorly sensitive to the dipole position (blue curves), the presence of the hybrid plasmonic nanostructures makes this dependence significant. This is likely to be due to the rich angular spectrum associated to the different plasmonic nanostructures, resulting in a strong gradient of the optical near-field along the $\mathrm{z}$ axis. In conclusion, Figure 4(c) shows that each dipole within the emissive layer can take advantage of specific plasmonic nanostructures, depending on its orientation and spatial position.

In a subsequent step, mechanical properties of the hybrid OLED was considered. EG layers were deposited on top of the AgNWs: AgNPs network with spray-coating. In this work, EG was used to enhance the mechanical flexibility of FTEs. Compared to the commonly used CVD-grown graphene, ${ }^{[46]}$ EG possesses the advantage of a low-cost and solution-processing, which is a better choice for the application of OLEDs. The uniformity of the deposition was deduced from the SEM and AFM observations. As shown in the bottom right part of Figure 5(a), when the AgNWs:AgNPs network is not covered with the EG layers, the AgNWs appear much brighter in the SEM image, clearly indicating the absence of EG on top of the AgNWs: AgNPs. Similarly, examination of the roughness in the AFM images provided a good insight into the presence or not of the EG layers. While the root-mean-square (RMS) of the topography of the AgNWs:AgNPs deposited on the PET substrate was $22.3 \mathrm{~nm}$, it significantly reduced to $7.6 \mathrm{~nm}$ after coating the AgNWs:AgNPs networks with the EG layers, as shown in Figure 5(b). Such a good uniformity is essential to contact the organic emissive layers. Furthermore, the small density of defects in EG that is reflected by a small D peak $\left(\sim 1350 \mathrm{~cm}^{-1}\right)$ in comparison with an intense $\mathrm{G}$ peak $\left(\sim 1580 \mathrm{~cm}^{-1}\right)$ and a visible 2D peak $\left(\sim 2700 \mathrm{~cm}^{-1}\right)$ in the Raman spectrum of Figure 5(c), indicates the high structural quality of the EG layers. 

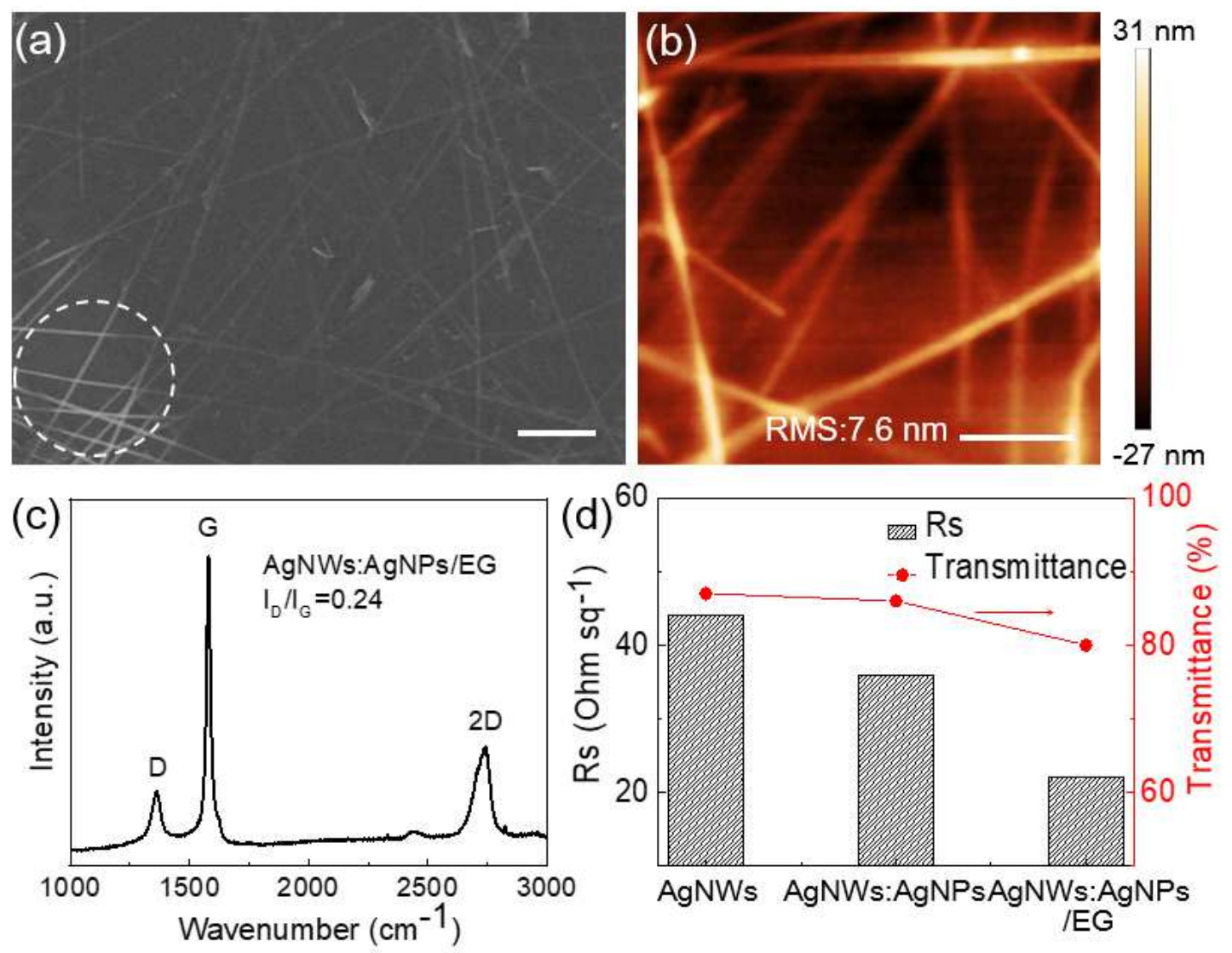

Figure 5. (a) SEM and (b) AFM images of EG deposition on AgNWs. The scale bar is $500 \mathrm{~nm}$. In (a), the dashed circle highlights AgNWs which are not covered by EG. (c) Raman spectra of AgNWs:AgNPs/EG film. (d) Optical transmittance and sheet resistance values of AgNWs, AgNWs:AgNPs and AgNWs:AgNPs/EG based FTEs.

It is known that there is an inverse correlation between sheet resistance $(R s)$ and optical transmittance of AgNW-based films, which are highly dependent on the fabrication process. ${ }^{[47]}$ Figure 5(d) shows the optical transmittance and $R s$ of the different nanostructured FTEs. After optimizing the density of AgNWs, the average transmittance of the FTE consisting of AgNWs only was 88\% over the whole visible spectral range, while $R_{s}$ was $44 \Omega \mathrm{sq}^{-1}$. When AgNPs were mixed into AgNWs, the average transmittance was slightly decreased to $86 \%$ due to screening effect by isolated AgNPs. But this decrease took along with a valuable reduction of $R_{s}$ to $36 \Omega \mathrm{sq}^{-1}$, which we attribute to the filling of empty spaces between the interconnected AgNWs by the AgNPs. This insertion of AgNPs improved the electrical contact between the NWs, making the carrier collection more efficient. After the coating of the AgNWs:AgN 
network with an uniform EG sheet, the transmittance dropped to $\sim 80 \%$. Such loss of transmittance with EG sheet indicates that the sheet contains a few (2 3) graphene layers. However, $R_{s}$ was further reduced to $22 \Omega \mathrm{sq}^{-1}$, due to an increase of the lateral conductivity since the empty gaps between AgNWs were bridged by the 2D EG layers. Finally, the figure of merit $(\mathrm{FoM})$ defined as the ratio of the electric conductivity to optical transparency, ${ }^{[48]}$ was used to better assess the performance of the FTEs. The calculations gave FoM values of 65, 67 and 75 for the FTEs consisting of AgNWs, AgNWs:AgNPs and AgNWs:AgNPs/EG, respectively.

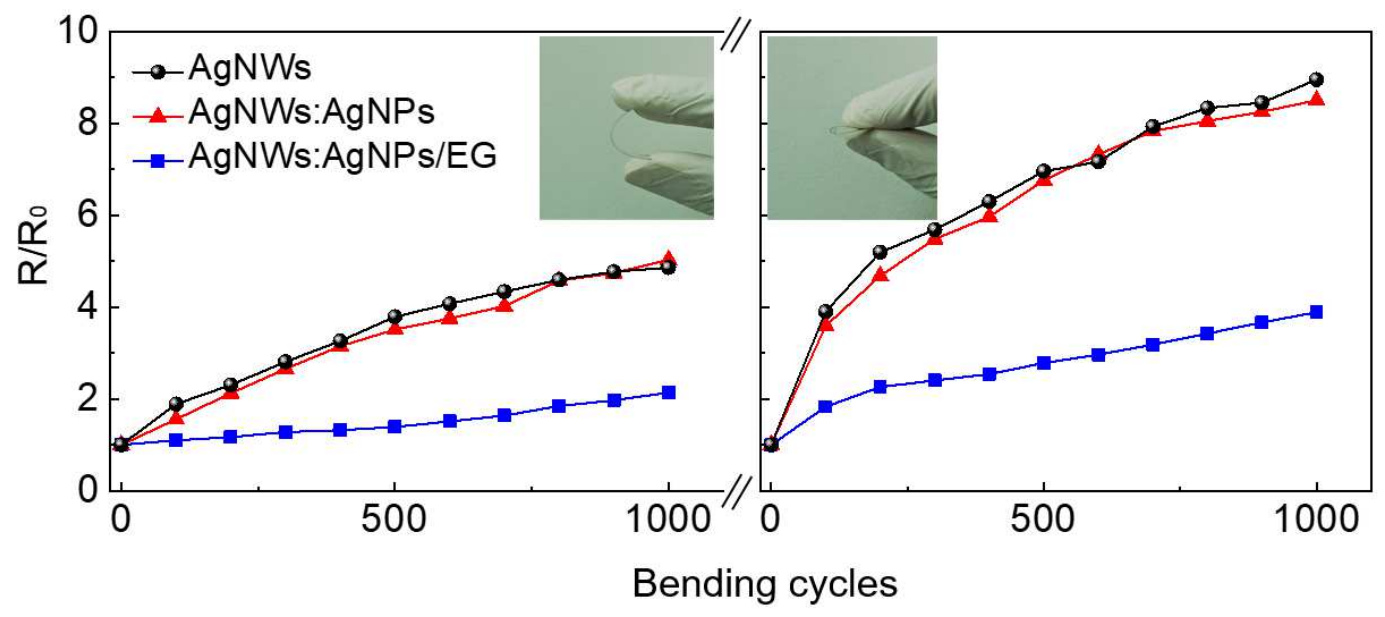

Figure 6. $R_{s} / R_{0}$ evolution as a function of bending (left) and folding (right) cycles for different FTEs of the bare AgNWs, AgNWs:AgNPs and AgNWs:AgNPs/EG. The radius of bending is $4 \mathrm{~mm}$. Insets are photographs of FTEs under bending and folding tests.

To investigate the mechanical flexibility of hybrid nanostructured FTEs induced by the coverage of EG layers, all three different electrodes were mechanically stressed. Figure 6 shows the variation of $R_{S}$ with respect to the initial value $R_{0}$ for $\mathrm{AgNWs}$, AgNWs:AgNPs and AgNWs:AgNPs/EG based FTEs during bending (left) and folding (right) tests. For a $4 \mathrm{~mm}$ bending radius, 1000 bending cycles yielded a ratio of $R_{s} / R_{0}$ that increased by a factor of 5 for AgNWs and AgNWs:AgNPs based FTE. When EG layers were added on the AgNWs:AgNPs network, the relatively small increase of $R_{s} / R_{0}$ by a factor of 2.1 shows an improved mechanical stability of the FTEs. Similarly, the $R_{S} / R_{0}$ value was dramatically increased to 8.5 and 8.9 for AgNWs:AgNPs and AgNWs 
based FTEs, respectively, after 1000 folding cycles with a radius lower than $1 \mathrm{~mm}$. In comparison, EG minimized the degradation of the conductivity with an increase of $R_{S} / R_{0}$ by a factor of 3.5 only, showing that the use of EG layers makes the FTEs more robust to mechanical stresses.

Finally, to assess the feasibility of the hybrid nanostructured plasmonic FTEs for application in flexible OLEDs, a set of flexible OLEDs were fabricated with a device architecture of PET/FTEs/PEDOT:PSS (40 nm)/ SY (60 nm)/Liq (1 nm)/Al (100 nm), as shown in Figure 7(a). PEDOT:PSS was used as hole transport layer, while SY was applied as the emissive layer. A bilayer cathode of Liq/Al was used for improving the electron collection. The control OLED with a device configuration of Glass/ITO/PEDOT:PSS (40 nm)/ SY (60 nm)/Liq (1 nm)/Al (100 nm) was also fabricated for comparison. The current density-voltage characteristics, luminance and current efficiency of OLEDs using ITO, AgNWs, AgNWs:AgNPs and AgNWs:AgNPs/EG FTEs are shown in Figure 6(b) and (c), while the device parameters are given in Table 1 .

The device with a AgNWs:AgNPs FTE showed substantially improved light-emitting characteristics with the luminance of $18010 \mathrm{~cd} \mathrm{~m}^{-2}$ at $10.5 \mathrm{~V}$ and current efficiency (CE) of $10.62 \mathrm{~cd} \mathrm{~A}^{-1}$ compared to the device with a AgNWs FTE (luminance of $13696 \mathrm{~cd} \mathrm{~m}^{-}$ ${ }^{2}$ at $11 \mathrm{~V}$ and $\mathrm{CE}$ of $8.55 \mathrm{~cd} \mathrm{~A}^{-1}$ ). The gain in luminance and $\mathrm{CE}$ is mainly attributed to the plasmon coupling of light induced by the addition of AgNPs, especially at the NWNP junctions. The device consisting of the AgNWs:AgNPs/EG FTE exhibited further improved performances (luminance of $20008 \mathrm{~cd} \mathrm{~m}^{-2}$ at $10.5 \mathrm{~V}$ and $\mathrm{CE}$ of $11.61 \mathrm{~cd} \mathrm{~A}^{-1}$ ), taking advantage of plasmonic effect of AgNWs:AgNPs and the high FoM value of FTEs induced by the 2D transport properties of EG layers. The performances of the latter devices with hybrid nanostructured plasmonic FTEs were found to be quite close to that using ITO (Figures 7(b) and (c)). Figure 7(d) shows the normalized luminance of OLEDs using AgNWs, AgNWs:AgNPs, AgNWs:AgNPs/EG FTEs and ITO electrodes after bending tests at a bending radius of $4 \mathrm{~mm}$, while Figure 7(e) shows the electroluminescence of the flexible OLEDs with AgNWs:AgNPs/EG FTEs under 
mechanical stress. The luminance of the devices with AgNWs, AgNWs:AgNPs and AgNWs:AgNPs/EG FTEs remained 61\%, 64\% and 90\% after 1000 bending, respectively. In contrast, the luminance of the ITO control device reduced dramatically to $42 \%$ of its initial value after only 100 bending cycles, demonstrating the clear advantage of our hybrid nanostructured plasmonic FTEs over the ITO electrode.
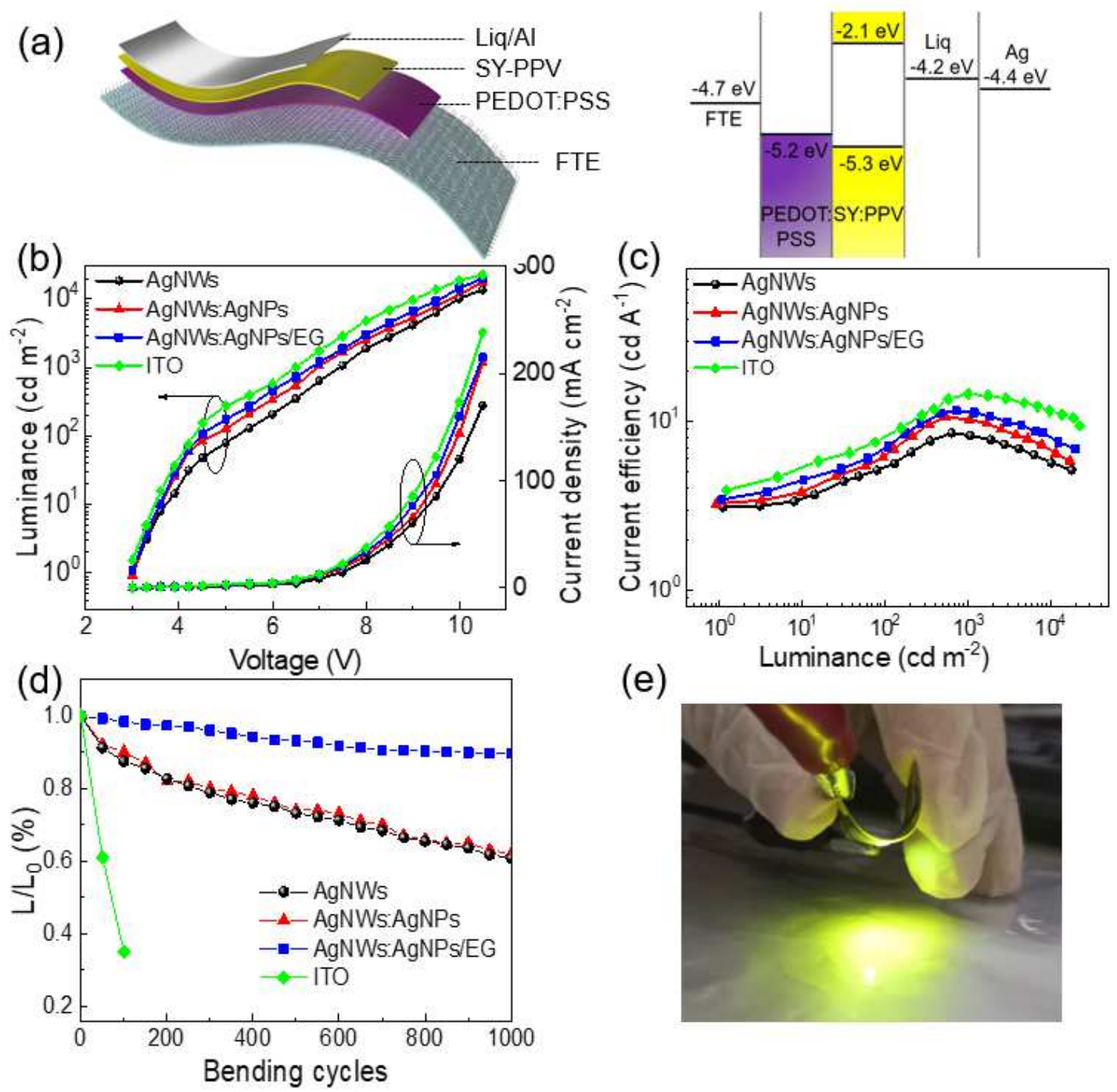

(e)

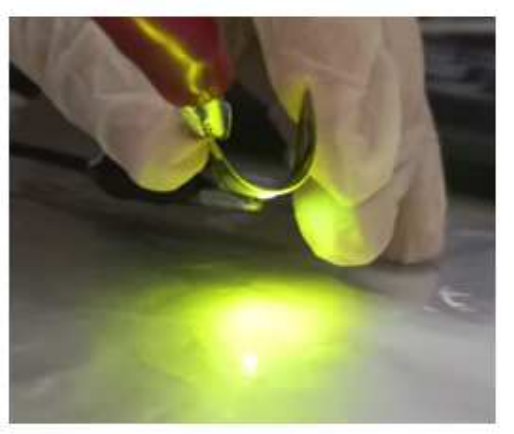

Figure 7. (a) Device structure of flexible OLEDs using hybrid nanostructured plasmonic FTEs and the corresponding energy level alignment. (b) Current density and Luminance plotted against voltage with AgNWs, AgNWs:AgNPs, AgNWs:AgNPs/EG FTEs and ITO electrodes. (c) Current efficiency plotted against luminance with AgNWs, AgNWs:AgNPs, AgNWs:AgNPs/EG FTEs and ITO electrodes. (d) Normalized luminance of OLEDs with AgNWs, AgNWs:AgNPs, AgNWs:AgNPs/EG FTEs and 
ITO electrodes after bending tests at a bending radius of $4 \mathrm{~mm}$. (e) Photograph of flexible OLEDs with AgNWs:AgNPs/EG based FTE under operation.

Table 1. Key parameters of OLEDs with AgNWs, AgNWs:AgNPs, AgNWs:AgNPs/EG FTEs and ITO electrodes.

\begin{tabular}{l|cccc}
\hline Types of electrodes & $\begin{array}{c}\mathrm{V}_{\text {on }} \\
(\mathrm{V})\end{array}$ & $\begin{array}{c}\mathrm{L}_{\max } \\
\left(\mathrm{cd} \mathrm{m}^{-2}\right)\end{array}$ & $\begin{array}{c}\mathrm{CE}_{\max } \\
\left(\mathrm{cd} \mathrm{A}^{-1}\right)\end{array}$ & $\begin{array}{c}\text { Current } \\
\text { Density } \\
\left(\mathrm{mA} / \mathrm{cm}^{2}\right)\end{array}$ \\
\hline ITO & 2.6 & 22498 & 14.92 & 238.6 \\
AgNWs & 3.1 & 13696 & 8.55 & 171.6 \\
Ag NWs:Ag NPs & 3.0 & 18010 & 10.62 & 210.3 \\
Ag NWs:Ag NPs/EG & 3.0 & 20008 & 11.61 & 215.4 \\
\hline
\end{tabular}

\section{Conclusion}

In this work, we have developed a solution processable hybrid nanostructured plasmonic electrode for use in flexible OLEDs. The synergy of OD AgNPs, 1D AgNWs and 2D exfoliated graphene sheets leads to a FTE with excellent electrical, optical and mechanical properties. In particular, the enhanced surface plasmonic effect of the NWNP hybrid plasmonic systems has been demonstrated by combining transient PL measurements with theoretical calculations, which leads to improved radiative emission in OLEDs. Flexible OLEDs with a current efficiency of $11.61 \mathrm{~cd} \mathrm{~A}^{-1}$ and a maximum luminance of $20008 \mathrm{~cd} \mathrm{~m}^{-2}$ were successfully fabricated with this hybrid FTE. Finally, the flexible OLEDs exhibit an excellent mechanical flexibility, retaining > 90\% of the initial luminance after a test of 1000 bending cycles. These results demonstrate that the hybrid nanostructured FTE with enhanced plasmonic effects is a favorable alternative to ITO for use in high-performance flexible OLEDs.

\section{Acknowledgements}

This work was financially supported by the National Natural Science Foundation 
of China (61775130, 11974236), Natural Science Foundation of Shanghai (19ZR1419500) and Science and Technology Commission of Shanghai Municipality Program (19DZ2281000).

\section{Reference}

[1] Zhang Z, Du J, Zhang D, Sun H, Yin L, Ma L and Ren W 2017 Rosin-enabled ultraclean and damage-free transfer of graphene for large-area flexible organic lightemitting diodes Nat. commun. 814560.

[2] Chen Z, Wang H, Wang X, Chen P, Liu Y, Zhao H and Duan Y 2017 Lowtemperature remote plasma enhanced atomic layer deposition of $\mathrm{ZrO}_{2}$ /zircone nanolaminate film for efficient encapsulation of flexible organic light-emitting diodes Sci. Rep, 740061.

[3] Kumar A and Zhou C 2010 The race to replace tin-doped indium oxide: which material will win? ACS. Nano 4(1) 11-14.

[4] Hecht D S, Hu L and Irvin G 2011 Emerging transparent electrodes based on thin films of carbon nanotubes, graphene, and metallic nanostructures Adv. Mater. 23(13) 1482-1513.

[5] Zhu Z, Mankowski T, Balakrishnan K, Shikoh A S, Touati F, Benammar M A and Falco C M 2015 Ultrahigh aspect ratio copper-nanowire-based hybrid transparent conductive electrodes with PEDOT: PSS and reduced graphene oxide exhibiting reduced surface roughness and improved stability ACS Appl. Mater. Inter. 7(30) 1622316230.

[6] Jin Y, Li L, Cheng Y, Kong L, Pei Q and Xiao F 2015 Cohesively enhanced conductivity and adhesion of flexible silver nanowire networks by biocompatible polymer sol-gel transition. Adv. Funct. Mater. 25(10) 1581-1587.

[7] Richardson B J, Zhu L and Yu Q 2017 Design and development of plasmonic nanostructured electrodes for ITO-free organic photovoltaic cells on rigid and highly flexible substrates Nanotechnology 28(16) 165401.

[8] Wang S L, Yang J, Xu T, Dou D, Tang Z, Gao Z, Chen M, Guo K, Yu J, Plain J, 
Bachelot R, Zhang J and Wei B 2019 Highly Efficient and Foldable Top-Emission Organic Light-emitting Diodes Based on Ag-Nanoparticles Modified Graphite Electrode Org. Electron. 64146.

[9] Wang S L, Zhao Y, Lian, H, Peng C, Yang X, Gao Y, Peng Y, Lan W, Ibrahim Elmi O, Stiévenard D, Wei B, Zhu F R and Xu T 2019 Towards All-solution-processed Topilluminated Flexible Organic Solar Cells using Ultrathin Ag-modified Graphite-coated Polyethylene Terephthalate Substrates. Nanophotonics. 8297.

[10] Ng M A, Hartadi L T, Tan H and Poa C P 2008 Efficient coating of transparent and conductive carbon nanotube thin films on plastic substrates Nanotechnology 19(20) 205703.

[11] Tung V C, Chen L M, Allen M J, Wassei J K, Nelson K, Kaner R B and Yang Y 2009 Low-temperature solution processing of graphene- carbon nanotube hybrid materials for high-performance transparent conductors Nano. Lett. 9(5) 1949-1955.

[12] Zhu X Z, Han Y Y, Liu Y, Ruan K Q, Xu M F, Wang Z K and Liao L S 2013 The application of single-layer graphene modified with solution-processed TiOx and PEDOT: PSS as a transparent conductive anode in organic light-emitting diodes Org. Electron. 14(12) 3348-3354.

[13] Krantz J, Stubhan T, Richter M, Spallek S, Litzov I, Matt G J and Brabec C J 2013 Spray-coated silver nanowires as top electrode layer in semitransparent P3HT: PCBMbased organic solar cell devices. Adv. Funct. Mater. 23(13) 1711-1717.

[14] Lee M H, Chen L X, Li N and Zhu F R 2017 MoO3-induced Oxidation Doping of PEDOT:PSS for High Performing Full-solution-processed Inverted Quantum-dot Light Emitting Diodes J. Mater. Chem. C 5 10555-10561.

[15] Ricciardulli A G, Yang S, Wetzelaer G J A, Feng X and Blom P W 2018 Hybrid Silver Nanowire and Graphene-Based Solution-Processed Transparent Electrode for Organic Optoelectronics. Adv. Func. Mater. 28(14) 1706010.

[16] Lei T, Peng R, Song W, Hong L, Huang J, Fei N and Ge Z 2019 Bendable and foldable flexible organic solar cells based on Ag nanowire films with 10.30\% efficiency. J. Mater. Chem A. 7(8) 3737-3744.

[17] Xu F and Zhu Y 2012 Highly conductive and stretchable silver nanowire conductors. Adv. Mater. 24(37) 5117-5122. 
[18] Wei B, Pan S, Wang T, Tian Z, Chen G and Xu T 2016 Solution-processed Agnanowire/ZnO-nanoparticle composite transparent electrode for flexible organic solar cells Nanotechnology. 27(50) 505208.

[19] Li L, Yu Z, Hu W, Chang C H, Chen Q and Pei Q 2011 Efficient flexible phosphorescent polymer light-emitting diodes based on silver nanowire-polymer composite electrode Adv. Mater. 23(46) 5563-5567.

[20] Selzer F, Wei N, Bormann L, Sachse C, Gaponik N, Müller-Meskamp L and Leo K 2014 Highly conductive silver nanowire networks by organic matrix assisted lowtemperature fusing Org. Electron. 15(12) 3818-3824.

[21] Lee Y, Suh M, Kim D, Lee D, Chang H, Lee H S and Jeon D Y 2014 Improved Operational Stability of Polymer Light-Emitting Diodes Based on Silver Nanowire Electrode Through Pre-Bias Conditioning Treatment Adv. Funct. Mater. 24(41) 64656472.

[22] Gaynor W, Burkhard G F, McGehee M D and Peumans P 2011 Smooth nanowire/polymer composite transparent electrodes Adv. Mater. 23(26) 2905-2910.

[23] Zeng X Y, Zhang Q K, Yu R M and Lu C Z 2010 A new transparent conductor: silver nanowire film buried at the surface of a transparent polymer Adv. Mater. 22(40) 4484-4488.

[24] Yu Z, Zhang Q, Li L, Chen Q, Niu X, Liu J and Pei Q 2011 Highly flexible silver nanowire electrodes for shape-memory polymer light-emitting diodes Adv. Mater. 23(5) 664-668.

[25] Zhang Q, Di Y, Huard C M, Guo L J, Wei J and Guo J 2015 Highly stable and stretchable graphene-polymer processed silver nanowires hybrid electrodes for flexible displays J. Mater. Chem. C. 3(7) 1528-1536.

[26] Hu L, Kim H S, Lee J Y, Peumans P and Cui Y 2010 Scalable coating and properties of transparent, flexible, silver nanowire electrodes ACS. Nano. 4(5) 2955-2963.

[27] Hardin B E, Gaynor W, Ding I K, Rim S B, Peumans P and McGehee M D 2011 Laminating solution-processed silver nanowire mesh electrodes onto solid-state dyesensitized solar cells Org. Electron. 12(6) 875-879.

[28] Xiong W, Liu H, Chen Y, Zheng M, Zhao Y, Kong X and Jiang L 2016 Highly Conductive, Air-Stable Silver Nanowire@ Iongel Composite Films toward Flexible 
Transparent Electrodes Adv. Mater. 28(33) 7167-7172.

[29] Liu Z Y, Wu Z S, Yang S, Dong R H, Feng X L and Mullen K 2016 Ultraflexible In-Plane Micro-Supercapacitors by Direct Printing of Solution-Processable Electrochemically Exfoliated Graphene Adv. Mater. 28 (11) 2217-2222.

[30] Ricciardulli A G, Yang S, Wetzelaer G A H, Feng X and Blom P W M 2018 Hybrid silver nanowire and graphene-based solution-processed transparent electrode for organic optoelectronics Adv. Funct. Mater. 281706010.

[31] Fujiki A, Uemura T, Zettsu N, Akai-Kasaya M, Saito A, Kuwahara Y 2010 Enhanced fluorescence by surface plasmon coupling of Au nanoparticles in an organic electroluminescence diode. Appl. Phys. Lett. 96043307.

[32] Kumar A, Srivastava R, Tyagi P, Mehta D, Kamalasanan M 2012 Efficiency enhancement of organic light emitting diode via surface energy transfer between exciton and surface plasmon. Org. Electron. 13159.

[33] Xiao Y, Yang J P, Cheng P P, Zhu J J, Xu Z Q, Deng Y H, Lee S T, Li Y Q, Tang J X 2012 Surface plasmon-enhanced electroluminescence in organic light-emitting diodes incorporating Au nanoparticles Appl. Phys. Lett. 100013308.

[34] Jeong S H, Choi H, Kim J Y and Lee T W 2015 Silver-Based Nanoparticles for Surface Plasmon Resonance in Organic Optoelectronics Part. Part. Syst. Char. 32(2) 164-175.

[35] Liu F and Nunzi J M 2011 Phosphorescent organic light emitting diode efficiency enhancement using functionalized silver nanoparticles Appl. Phys. Lett. 99(12) 203. [36] Kumar A, Srivastava R, Mehta D S and Kamalasanan M N 2012 Surface plasmon enhanced blue organic light emitting diode with nearly $100 \%$ fluorescence efficiency Org. Electron. 13(9) 1750-1755.

[37] Kim T, Kang S, Heo J, Cho S, Kim J W, Choe A and Kim J Y 2018 NanoparticleEnhanced Silver-Nanowire Plasmonic Electrodes for High-Performance Organic Optoelectronic Devices Adv Mater. 30(28) 1800659.

[38] Knight M W, Grady N K, Bardhan R, Hao F, Nordlander P and Halas N J 2007 Nanoparticle-mediated coupling of light into a nanowire Nano. Lett. 7(8) 2346-2350.

[39] Hao F, Nordlander P 2006 Plasmonic coupling between a metallic nanosphere and a thin metallic wire Appl. Phys. Lett. 89, 103101. 
[40] Akjouj A, Saison-Francioso O, Lévêque G, Boukherroub R, Szunerits S. 2015 Dependence Between the Refractive-Index Sensitivity of Metallic Nanoparticles and the Spectral Position of Their Localized Surface Plasmon Band: A Numerical and Analytical Study J. Phys. Chem. C 11928551.

[41] Johnson P B, Christy R W 1972 Optical Constants of the Noble Metals. Phys. Rev. B. $6,4370-4379$.

[42] Barnes W L, Dereux A, Ebbesen T W 2003 Surface plasmon subwavelength optics Nature 424, 824.

[43] Choi H, Ko S J, Choi Y, Joo P, Kim T, Lee B R and Hwang I W 2013 Versatile surface plasmon resonance of carbon-dot-supported silver nanoparticles in polymer optoelectronic devices Nat. Photonics 7(9) 732.

[44] Ji W, Zhao H, Yang H and Zhu F R 2015 Effect of coupling between excitons and gold nanoparticle surface plasmons on emission behavior of phosphorescent organic light-emitting diodes Org. Electron. 22154.

[45] Jung D R, Kim J, Nam S, Nahm C, Choi H, Kim J I and Park B 2011 Photoluminescence enhancement in $\mathrm{CdS}$ nanoparticles by surface-plasmon resonance Appl. Phys. Lett. 99(4) 041906.

[46] Xu T, Díaz Álvarez A, Wei W, Eschimese D, Eliet S, Lancry O, Galopin E, Vaurette F, Berthe M, Wei B, Xu J, Lampin J F, Pallecchi E, Happy H, Vignaud D and Grandidier B 2018 Transport mechanisms in a puckered graphene-on-lattice Nanoscale. 107519. [47] Zhang Y X, Fang J, Li W, Shen Y, Chen J D, Li Y Q, Gu H W, Pelivani S, Zhang M J, Li Y F and Tang J X 2019 Synergetic Transparent Electrode Architecture for Efficient Non-Fullerene Flexible Organic Solar Cells with >12\% Efficiency ACS Nano 13 4686-4694.

[48] Xu T, Gong C, Wang S L, Lian H, Lan W, Lévêque G, Grandidier B, Plain J, Bachelot R, Wei B and Zhu F R 2020 Ultraviolet durable flexible nonfullerene organic solar cells realized by a hybrid nanostructured transparent electrode. Solar RRL. 1900522. 